SLAC - PUB - 3916

April 1986

$\mathrm{T} / \mathrm{E}$

\title{
COMMENT IN REPLY*
}

\author{
H. PierRe Noyes \\ Stanford Linear Accelerator Center \\ Stanford University, Stanford, California, 94905
}

\begin{abstract}
This reply claims to show that the mathematical difficulty noted by Orlowski was already met in the original paper by invoking the physical restrictions arising from relativistic kinematics and an ordered limit based on them. In contrast we claim that Orlowski's procedure is unmotivated physically, ad hoc from a mathematical point of view, and, even if adopted, too flexible to be of much use in most problems of physical interest.
\end{abstract}

Submitted to Physical Review $C$ in reply to a Comment by M.K.Orlowski on the paper

H.Pierre Noyes Phys.Rev. C 26,1858-77(1982)

* Work supported by the Department of Energy, contract DE - AC03-76SF00515. 
In his comment ${ }^{1}$ on my ${ }^{\| 1}$ paper $^{2}$ Orlowski has failed to understand what I was trying to communicate in two significant ways. I reply because the physics and the mathematics are intertwined in a way that is not superficial; I hope that reading his exposition and mine together may enable the reader to understand the differences; from my point of view these differences are errors on his part and not just misunderstandings.

Orlowski is correct in stating that the zero range theories as usually encountered in the literature "suffer from divergences and do not lead to finite results for three-body observables." This was already noted in my paper in the fifth paragraph of the introduction ${ }^{3}$. This fact was proved by L.H.Thomas in 1935 (Ref. 8 of ZRST.I.) and subsequently given more generality (Ref. 9 of ZRST.I, also cited by Orlowski). I also agree with Orlowski that the prescriptions used to obtain finite results as given in these references are ad hoc. However, the divergences associated with the "zero range limit" are physically meaningless because they refer to an energy region where the non-relativistic approximation is itself meaningless. Clearly a cutoff is required; the problem is to make it meaningful rather than ad hoc.

My approach, as was briefly discussed, is to insist that any nonrelativistic theory becomes physically meaningless when the energies approach the rest energies of the particles. Brayshaw had shown that the relativistic kinematics itself Lisuices the finite range boundary condition model deliver finite answers for the three particle binding energies no matter how small the range is. Lindesay had shown that a simple covariant model, whose on-shell amplitude reduces to the

H1 Since these are single author papers, I use for clarity in exposition " $I$ " and "he" rather than the more formal "we" and "they". 
scattering length model in the nonrelativistic limit, not only provides finite and unitary amplitudes in the relativistic environment (which never need be abandoned) but also quantitatively reproduces (in the appropriate parametric region) phenomena previously only derived non-relativistically (the Efimov effect) ${ }^{4}$.

Having showed that relativity introduces a cutoff into theories which approach the non-relativistic zero range limit, and which is required for such theories to make sense, we did not think it necessary in the paper to explore the parameter range allowed to that cutoff. If it was above the meson mass measured in units of the nucleon mass (where the non-relativistic model already becomes suspect) any prescription giving stable results in the range from there up to the nucleon mass will do. The divergence Orlowski refers to lies beyond that range, and even there is only logarithmic in the ratio of the cutoff to the nucleon mass.

The mathematical point which Orlowski misses is that once any cutoff has made the Faddeev Equations

$$
\begin{gathered}
M_{a b}(z)-t_{a}(z) \delta_{a b} \\
=-t_{a}(z) R_{0}(z)\left[M_{b b}(z)+M_{c b}(z)\right]=-\left[M_{a a}(z)+M_{a c}(z)\right] R_{0}(z) t_{b}(z)
\end{gathered}
$$

finite, the zero range model allows the two particle amplitudes to be factored out of the integrals by defining

$$
M_{a b}(z)-t_{a}(z) \delta_{a b}=t_{a}(z) Z_{a b}(z) t_{b}(z)
$$

We then find that the equations

$$
Z_{a b}(z)+\bar{\delta}_{a b} R_{0}(z)
$$




$$
=-R_{0}(z)\left[t_{b}(z) Z_{b b}(z)+t_{c}(z) Z_{c b}(z)\right]=-\left[Z_{a a}(z) t_{a}(z)+Z_{a c}(z) t_{c}(z)\right] R_{0}(z)
$$

are convergent even for input which would produce divergences in the $M_{a b}$ equations. Hence we can from now on ignore the cutoff for any reasonable application of the theory presented in ZRST.I. All we need insure is that the representation of the $t_{a}$ has requisite accuracy (i.e. reproduces nucleon-nucleon phase shifts within experimental error below meson production threshold) and that the three body results are insensitive to how the cutoff is made at energies above the nucleon mass. In a mathematical sense, the divergences to which Orlowski refers have been unambiguously banished from the theory. Of course, for internal consistency in terms of the representation of phase shifts by analytic functions, these can have no "left hand cuts" for the reasons spelled out in ZRST.I. But since we need represent the phase shifts only to finite accuracy over a finite range of energies, known in advance, this is no problem.

In contrast, as Orlowski admits, his formalism is discontinuous in the limit we both seek, and can only be used because of a "plateau" - which he exhibits only numerically and does not investigate mathematically. It is his prescription which is correctly characterized as ad hoc; we do not see any easy way to give it precision. So much for the first "error" or misunderstanding.

Orlowski's second departure from our way of thinking is even more fundamental. He approaches the problem by introducing an energy-dependent separable interaction in such a way that the parameters of the separable interaction can be adjusted to two-nucleon data (phase shifts and binding energies) without affecting the off shell (finite range) parameters; as already noted this off shell behavior cannot be taken to an exact zero range limit without reintroducing the zero range singularity. My second criticism of his approach is an old one: his theory falls 
into a class of non-relativistic modeling approaches which are too flexible. They do not provide any clear relationship between their parameters and the physical information needed to solve the three nucleon problem ${ }^{5}$. Further, if the parameters in the model are used to fit three-nucleon data, they must remain silent when posed with the question as to why the data are fitted this way rather than by introducing a "three body force" ${ }^{6}$. Thus, while Orlowski's model may be interesting for someone who wants a unitary way to fit two and three body data, and for those so inclined I can hardly object to it, I must protest against his use of the term "physical" either for his model or for his "zero range approximation". We both agree that the formal mathematical limit is meaningless in his theory. In mine I claim that my ordered limit, based throughout on sound physical principles, is well defined mathematically as well.

By sticking to the physics, Lindesay and I have constructed a fully relativistic model which starts from a covariantly defined "zero range" input (an "s-channel pole" without structure) and in the two-nucleon one meson problem leads to a unitary two nucleon scattering amplitude characterized by a meson mass and coupling constant ${ }^{7}$. When the two-nucleon off-shell amplitude calculated by solving the two-nucleon one-meson problem is used in the three-nucleon problem, this model gives well defined relativistic Faddeev equations. When the same model is used to formulate the three-nucleon one-meson problem directly, the resulting Faddeev-Yakubovsky equations reduce to the same result ${ }^{8}$. In effect we have given a relativistic definition of the "potential" due to single meson exchange. Further, since the internal mesonic degree of freedom is completely Specified, the interaction of the system with electromagnetic fields, or quanta, can be readily calculated. Since we have completely determined the relativistic kine- 
matic effects without producing "three body forces", comparison with treatments containing more internal degrees of freedom (pion-nucleon resonances, pion-pion scattering,anti-nucleons, quarks and gluons,...) should allow an unambiguous separation of physical three nucleon forces from the hitherto ambiguous contributions arising from relativistic kinematic effects.

\section{REFERENCES}

1. M.K.Orlowski,Comment above.

2. H.P.Noyes, Phys. Rev. C 26, 1858-1877(1982), hereinafter referred to as ZRST.I.

3. Ref. $2,1^{\text {st }}$ column, starting at line 17 .

4. James V. Lindesay, PhD Thesis, Stanford, 1981; SLAC Report No. 243.

5. H.P.Noyes, "Physical Information Needed to Solve the Three-Nucleon Problem", Progress in Nuclear Physic, 10, 355-380 (1968).

6. H.P.Noyes, “Three Body Forces”, in Few Body Problems, I.Slaus, et.al. eds, North Holland, Amsterdam, 1972, p.122.

7. H.P.Noyes and J.V.Lindesay, Australian J. Physics, 36, 601 (1983).

8. H.P.Noyes, "A Minimal Relativistic Model for the Three Nucleon System", submitted to the International Conference on Three Body Forces in the Three Nucleon System, George Washington University, Washington D.C., April 24-26, 1986, included here as the Appendix. 


\section{APPENDIX:A MINIMAL RELATIVISTIC MODEL FOR THE THREE NUCLEON SYSTEM*}

The difficulty in discussing "three body forces" in the three nucleon system stems from the fact that these arise from "hidden" degrees of freedom due meson exchange, and presumably at some level of accuracy due to the quarkgluon substructure. Yet relativistic theories, whether from a field-theoretic or a dispersion-theoretic point of view seem to require the introduction of an infinite number of degrees of freedom with no generally valid method for bringing these down to negotiable, finite terms for the problem at hand.

A first step toward the resolution of this dilemma has been taken recently by the development of a complete relativistic scattering theory containing only three particulate degrees of freedom ${ }^{1}$. Unfortunately the very generality of the treatment obscures the basic simplifications this approach makes possible. Here we use a model in which the three body theory is driven by a simple "s-channel pole" in the two-body input scattering amplitudes". Restricted for simplicity to scalar nucleons and mesons, this gives a simple relativistic model for the nuclear force obtained by allowing the only input poles to occur in the mesonnucleon channcls at the mass of the nucleon, thus prohibiting direct nucleonnucleon scattering ${ }^{3}$. This relativistic treatment of the two nucleon-one meson system allows only two nucleons to appear asymptotically (i.e. in the laboratory) while retaining the mesonic degree of freedom in the internal dynamics. This system can be extended to the three nucleon system in two ways ${ }^{4}$. In the first one uses the fully defined off shell two-nucleon amplitude calculated from the two nucleon-one meson equations as input for the $(3 \times 3)$ Faddeev amplitudes it generates in relativistic three particle equations; in the second one applies the same model to the three nucleon-one meson system and calculates the FaddeevYakubovsky amplitudes. The simple treatment of the relativistic kinematics that our "confined quantum" approach allows when we re-express the equations in Mandelstam variables ${ }^{5}$ reduces the four body problem to three initial and three final channels (determined by which one of the three particles includes the meson) and makes the two treatments identical, as was originally conjectured by Sawicki.

For three scalar particles of mass $m_{a}, m_{b}, m_{c}$, the Faddeev equations for

our model are $-M_{a b}+\frac{g_{a} g_{b}}{s_{a}-8_{a}^{0}} \delta_{a b}=\frac{g_{a}^{2}}{s_{a}-8_{a}^{0}} R_{0}\left(M_{c b}+M_{b b}\right)=\left(M_{a a}+M_{a c}\right) R_{0} \frac{g_{b}^{2}}{s_{b}-s_{b}^{0}}$ where $s_{a}$ is the square of the invariant four momentum of the $b c$ pair and $R_{0}$

$\# 1$ Submitted to the International Conference on Three Body Forces in the Three Nucleon System, George Washington University, Washington D.C., April 24-26, 1986. 
the relativistic propagator. If we define $M_{a b}-\frac{g_{a} g_{b}}{s_{a}-8_{a}^{0}} \delta_{a b}=\frac{g_{a}}{s_{a}-s_{a}^{0}} H_{a b} \frac{g_{b}}{s_{b}-s_{b}^{0}}$, and $V_{a b}=-\frac{g_{a}}{s_{a}-s_{a}^{0}} R_{0} \bar{\delta}_{a b} \frac{g_{b}}{s_{b}-s_{b}^{0}}$ it follows immediately that $H_{a b}=V_{a b}-\frac{V_{a c} H_{a b}}{s_{c}-s_{c}^{0}}-\frac{V_{a b} H_{b b}}{s_{b}-s_{b}^{0}}=$ $V_{a b}-\frac{H_{a b} V_{a b}}{s_{a}-s_{a}^{o}}-\frac{H_{a c} V_{a b}}{s_{c}-s_{c}^{o}}$ Since the elastic and rearrangement amplitudes satisfy Lippmann-Schwinger equations for a three-channel system with a time-reversal invariant "potential" they are guaranteed to be unitary independent of the choice of coupling constants. For our nuclear force model we take $m_{a}=m_{b}=m$, $m_{c}=\mu, g_{a}=g_{b}=g$ and $g_{c}=0$ and, after symmetrization find that for nonrelativistic kinematics we have the sum of an ordinary and exchange Yukawa potential. Including nuclear isospin, this "Serber force" is not a bad zero" ${ }^{\text {th }}$ approximation for the nuclear force. Since the three nucleon system can now be calculated either from the pair-wise "potentials" or from the two-body unitary amplitudes, our solutions for the three nucleon-one meson system are unitary.

A number of interesting applications follow. In effect we have given a relativistic definition of the "potential" due to single meson exchange. Further, since the internal mesonic degree of freedom is completely specified, the interaction of the system with electromagnetic fields, or quanta, can be readily calculated. Since we have completely determined the relativistic kinematic effects without producing "three body forces", comparison with treatments containing more internal degrees of freedom (pion-nucleon resonances, pion-pion scattering,anti-nucleons, quarks and gluons,...) should allow an unambiguous separation of physical three nucleon forces from the hitherto ambiguous contributions arising from relativistic kinematic effects.

\section{REFERENCES}

1. J.V.Lindesay, A.J.Markevich, H.P.Noyes \& G.Pastrana, and Alexander J. Markevich, Phys.Rev.D (April 15, 1986).

2. James V. Lindesay, PhD Thesis, Stanford, 1981; SLAC Report No. 243.

3. H.P.Noyes and J.V.Lindesay, Australian J. Physics, 36, 601 (1983).

4. H.P.Noyes and M.K.Orlowski, Nuovo Cimento 81 A, 617 (1984).

5. G.Pastrana, $\mathrm{PhD}$ thesis, Stanford (in preparation). 